Making ICRF power compatible with a high-Z wall in ASDEX Upgrade

This content has been downloaded from IOPscience. Please scroll down to see the full text.

2017 Plasma Phys. Control. Fusion 59014022

(http://iopscience.iop.org/0741-3335/59/1/014022)

View the table of contents for this issue, or go to the journal homepage for more

Download details:

IP Address: 157.193.64.83

This content was downloaded on 04/05/2017 at 10:06

Please note that terms and conditions apply.

You may also be interested in:

First results with 3-strap ICRF antennas in ASDEX Upgrade

V. Bobkov, F. Braun, R. Dux et al.

ICRF operation with improved antennas in ASDEX Upgrade with $\mathrm{W}$ wall

V. Bobkov, M. Balden, R. Bilato et al.

Assessment of compatibility of ICRF antenna

VI.V. Bobkov, F. Braun, R. Dux et al.

Spatial proximity effects on the excitation of sheath RF voltages by evanescent slow waves in the ion cyclotron range of frequencies

Laurent Colas, Ling-Feng Lu, Alena Kivská et al.

Reduction of RF-sheaths potentials by compensation or suppression of parallel RF currents on ICRF antennas

A. Mendes, L. Colas, K. Vulliez et al.

Optimization of ICRH for core impurity control in JET-ILW

E. Lerche, M. Goniche, P. Jacquet et al.

Hot spot phenomena on Tore Supra ICRF antennas

L. Colas, L. Costanzo, C. Desgranges et al.

Understanding the spatial structure of RF-induced SOL modifications

L Colas, A Ekedahl, M Goniche et al. 


\title{
Making ICRF power compatible with a high-Z wall in ASDEX Upgrade
}

\author{
V Bobkov ${ }^{1}$, D Aguiam ${ }^{2}$, R Bilato ${ }^{1}$, S Brezinsek ${ }^{3}$, L Colas $^{4}$, H Faugel $^{1}$, \\ H Fünfgelder ${ }^{1}$, A Herrmann ${ }^{1}$, J Jacquot ${ }^{1}$, A Kallenbach ${ }^{1}$, D Milanesio ${ }^{5}$, \\ R Maggiora ${ }^{5}$, R Neu ${ }^{1,6}$, J-M Noterdaeme ${ }^{1,7}, \mathbf{R}$ Ochoukov $^{1}$, S Potzel ${ }^{1}$, \\ T Pütterich ${ }^{1}$, A Silva ${ }^{2}$, W Tierens ${ }^{1}$, A Tuccilo ${ }^{8}$, O Tudisco $^{8}$, Y Wang $^{9}$, \\ $Q$ Yang ${ }^{9}$, W Zhang ${ }^{1}$ and ASDEX Upgrade Team \\ and the EUROfusion MST1 Team ${ }^{10}$ \\ 1 Max-Planck-Institut für Plasmaphysik, Boltzmannstr. 2, 85748 Garching, Germany \\ 2 Instituto de Plasmas e Fusão Nuclear, Instituto Superior Técnico, Universidade de Lisboa, \\ 1049-001 Lisboa, Portugal \\ 3 Forschungszentrum Jülich GmbH, Institut für Energie- und Klimaforschung, Plasmaphysik, \\ Partner of the Trilateral Euregio Cluster (TEC), 52425 Jülich, Germany \\ ${ }^{4}$ IRFM, Association EUROFUSION-CEA, Saint-Paul-Lez-Durance, France \\ 5 Politecnico di Torino, Torino, Italy \\ 6 Technische Universität München, Boltzmannstr. 15, 85748 Garching, Germany \\ 7 Applied Physics Department, University of Ghent, Ghent, Belgium \\ ${ }^{8}$ ENEA, Frascati, Italy \\ 9 ASIPP, Institute of Plasma Physics, Chinese Academy of Sciences, Hefei, People's Republic of China \\ E-mail: bobkov@ipp.mpg.de
}

Received 1 July 2016, revised 29 August 2016

Accepted for publication 13 September 2016

Published 27 October 2016

\begin{abstract}
A comparison of the ASDEX Upgrade 3-strap ICRF antenna data with the linear electromagnetic TOPICA calculations is presented. The comparison substantiates a reduction of the local electric field at the radially protruding plasma-facing elements of the antenna as a relevant approach for minimizing tungsten (W) sputtering in conditions when the slow wave is strongly evanescent. The measured reaction of the time-averaged RF current at the antenna limiters to the antenna feeding variations is less sensitive than predicted by the calculations. This is likely to have been caused by temporal and spatial fluctuations in the 3D plasma density distribution affected by local non-linear interactions. The 3-strap antenna with the W-coated limiters produces drastically less W sputtering compared to the W-coated 2-strap antennas. This is consistent with the non-linear asymptotic SSWICH-SW calculations for RF sheaths.
\end{abstract}

Keywords: ICRF, RF sheath, antenna, W sputtering, TOPICA, SSWICH, ASDEX Upgrade

(Some figures may appear in colour only in the online journal)

\section{Introduction}

The operation of ion cyclotron range of frequency (ICRF) antennas is often accompanied by parasitic processes such as impurity production and additional heat loads on the plasmafacing components (PFCs). These are likely to be the consequence of RF-induced electrical fields near the antenna-in

\footnotetext{
${ }^{10}$ See http://www.euro-fusionscipub.org/mst1
}

particular the parallel field $E_{\|}$. The latter is responsible for the formation of RF sheaths by driving more mobile electrons to the antenna PFCs and charging up the plasma, as the PFCs are usually grounded. The RF sheaths are characterized by an elevated time-averaged or a DC potential drop close to the conductive surfaces. This leads to enhanced physical sputtering by accelerated ions and to increased heat loads.

In the all-tungsten (W) ASDEX Upgrade (AUG), ICRFspecific $\mathrm{W}$ production had a strong influence on the ICRF 


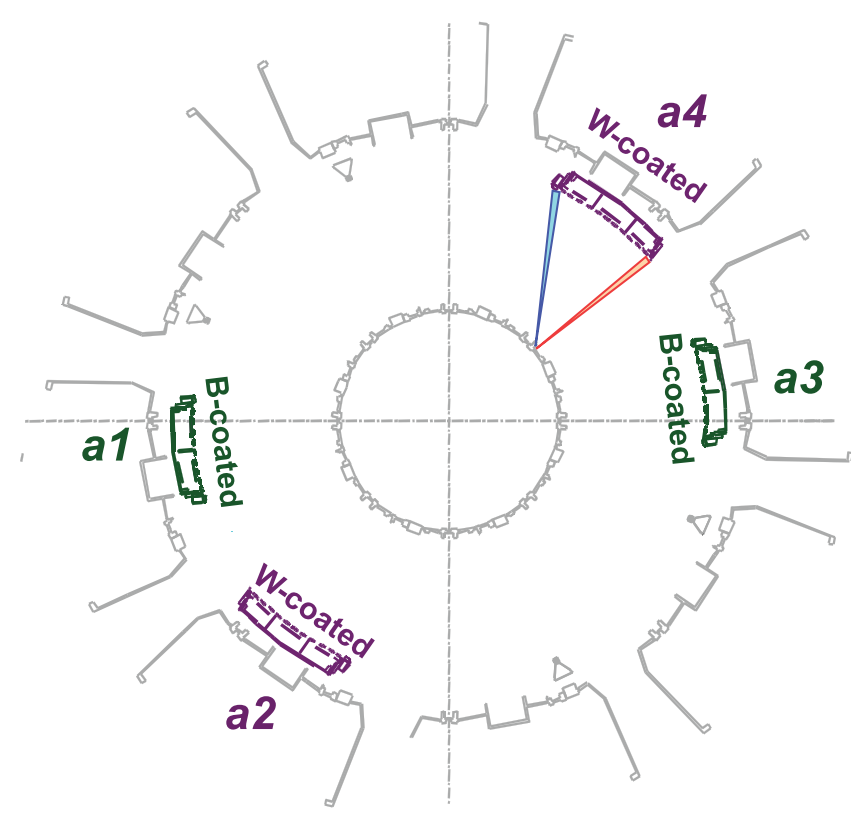

Figure 1. A toroidal view of the ICRF antennas and spectroscopy views at $a 4$ in the ASDEX Upgrade.

applicability due to increased radiation [1]. Some aid to reducing $\mathrm{W}$ production was found in tailoring the plasma properties in the scrape-off layer (SOL) by shifting the plasma radially away from the antenna [2] and by local gas injection [3]. Nevertheless, it was the installation of the boron (B)-coated limiters at the 2-strap antennas that enlarged the ICRF operational window in the AUG significantly. However, the root cause of the enhanced sputtering was not removed by the use of B-limiters. The sputtering remained, but the increased content of the low-radiating low- $Z$ boron became more tolerable than that of the high-Z tungsten. In parallel, antenna optimization was studied. As a first step, one of the 2-strap antennas was modified with broad-limiters and optimized straps which showed up to a $40 \%$ reduction in $\mathrm{W}$ release [3]. A second, more advanced step was the installation of new 3 -strap antennas [3] in 2015, and this constitutes the main subject of the paper. The corresponding arrangement of the ICRF antennas in the ASDEX Upgrade is presented in figure 1 with two B-coated 2-strap antennas $(a 1, a 3)$ and two 3-strap W-coated antennas $(a 2, a 4)$.

In section 1 , the paper discusses slow wave propagation in the private antenna region and the simplified approach for minimizing RF sheath effects by optimization of the near-field distribution. Section 2 is devoted to a description of the measurements, and a comparison of the calculations and relations to the $\mathrm{W}$ sputtering. Section 3 summarizes the total effect of 3 -strap antennas on the reduction of $\mathrm{W}$ release.

\section{Minimization of RF sheath effects}

Over the last decade, experimental activities in many machines with ICRF heating have allowed the irrelevance of the previous approach often used to minimize RF sheaths to be pinpointed; this is discussed below in section 1.1. The assessment of slow wave propagation in section 1.1 allows us to substantiate a simplified relevant approach. We apply this approach in section 1.2, when considering the $E_{\|}$-distribution at the 3-strap antenna.

\subsection{Relevance of the approach for minimizing the effect of RF sheaths}

In the past, in order to characterize the effect of RF nearfields on antenna-plasma interactions due to RF sheaths, the approach of calculating the integral of $E_{\|}$along a magnetic field line in the absence of the sheaths at the plasma-wall boundaries was often used. The so-called sheath-driving RF voltage [4], $V_{\mathrm{RF} \text { sheath }}=\left|\int E_{||}(\vec{l}) \cdot \mathrm{d} \vec{l}\right|$ was estimated as a figure of merit to minimize antenna-plasma interactions (see e.g. [5]). This basic linear approach was simple to use, but questionable from many points of view. Firstly, in contrast to [5], in many cases only the long field lines were considered. These pass in front of the antenna [6,7] without intersecting any conducting structure and are spatially limited just by the calculation frame. The calculated RF voltage was supposed to be relevant for local RF sheath formation deep in the private plasma region of an antenna. However, the result could be strongly influenced by the RF field excited in remote locations not connected to this private region along the magnetic field lines. Secondly, the direct integration of $E_{\|}$implied perfect parallel plasma conductivity. Thus, it was not taken into account that on an RF timescale the conducting properties of the plasma are typically dominated by plasma polarization effects and displacement currents. The use of this approach to minimize the ICRF-specific heat loads at the Tore Supra antenna [6] resulted in the absence of such minimization after the Faraday screen (FS) was modified [8]. The figure of merit described above was also inconsistent with the experimental observations in monopole phasing at Alcator C-Mod [7, 9]. Recent experimental observations of heat flux in antenna components in Tore Supra [8] and of the RF image current on the frame of the imbalanced 2-strap antenna in the ASDEX Upgrade [10] showed strong asymmetries at opposite ends of the field lines. This means that the quantities relevant for antenna-plasma interactions cannot be described by a single value for a given magnetic field line.

For the AUG antenna improvement, a different approach using the local values of $E_{\|}$as a figure of merit was advocated in $[2,3,11]$. A reduction in the local $E_{\|}$-field values close to the radially protruding antenna side limiters, i.e. the conducting structures, was targeted in particular. Here, magnetic field lines intersect the conducting surfaces and the high $E_{\|}$-field can be excited if local RF currents exist. The presence of radially protruding structures also increases electron losses with the resulting formation of RF sheaths. Far away from these structures, along the magnetic field lines, electrons can show the oscillatory behaviour imposed by the RF cycle. However, the influence of remote RF field excitation due to wave propagation was not taken into account in this approach. Recent work with the SSWICH-SW code [12, 13] and an analytic formulation of slow wave propagation and sheath boundary conditions [14] allow for further substantiation of the AUG approach. In [14], the weighted integration of $E_{\|}$along the 


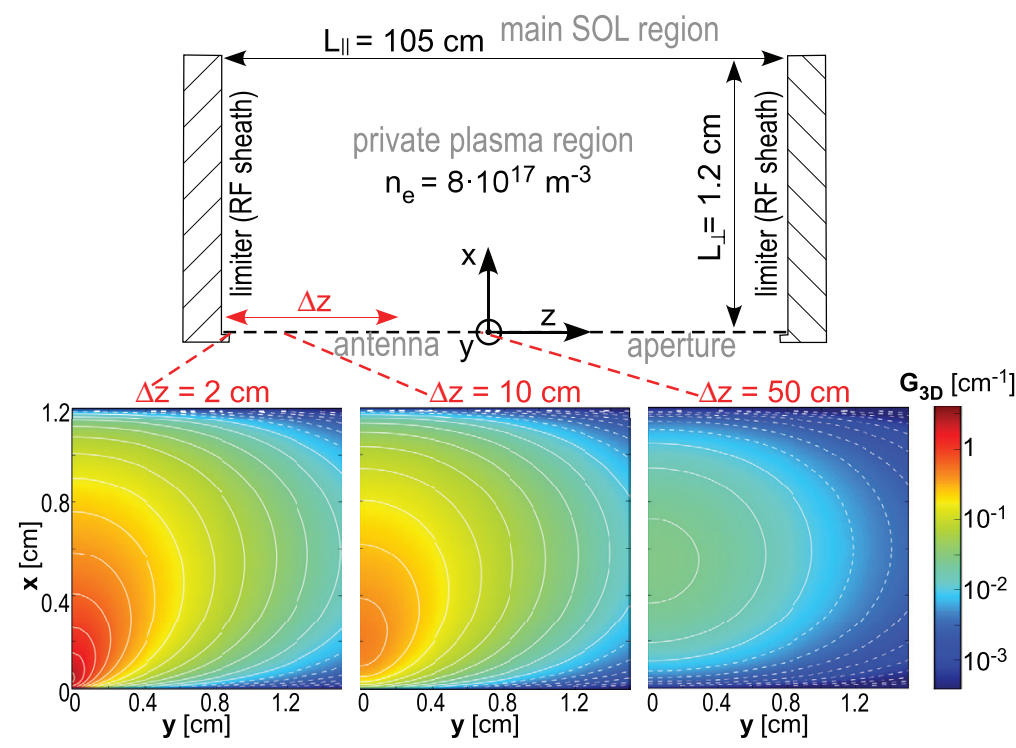

Figure 2. The geometry of calculations and the weighting factor $G_{3 \mathrm{D}}$ versus the transverse coordinates $(x, y)$, as evaluated numerically using parallel distances $\Delta z=2 \mathrm{~cm}, \Delta z=10 \mathrm{~cm}$ and $\Delta z=50 \mathrm{~cm}$.

field lines $V_{\mathrm{RF} \text { sheath }}(\vec{r})=\int G\left(\vec{r}, \vec{r}_{0}\right) E_{||}\left(\vec{r}_{0}\right) \cdot \mathrm{d} \vec{r}_{0}$ is proposed, in order to take into account the propagation of the slow wave in the presence of the sheaths at the plasma-wall boundaries. For the simple geometry presented in figure 2, this integration would be made along the parallel axis $z$. In general, $G\left(\vec{r}, \vec{r}_{0}\right)$ is the weighting function which depends on the position of the sheath boundary $\vec{r}$ (in the AUG case, the limiters) for which the sheath oscillating voltage $V_{\mathrm{RF}}$ sheath is calculated and on the position $\vec{r}_{0}$ of every $E_{\|}$wave emitting point on the aperture of the antenna (typically a toroidal-poloidal plane just in front of the antenna). The slow wave is assumed to be the dominant contributor to $E_{\|}$. The function $G\left(\vec{r}, \vec{r}_{0}\right)$ describes how the slow wave transmits the $E_{\|}$-field from its source to a point at the limiters where an RF sheath is formed. If this transmission is weak over a characteristic length of the antenna, the RF sheath is influenced by the local $E_{\|}$only.

We estimate the slow wave transmission of the $E_{\|}$-field by calculating $G\left(\vec{r}, \vec{r}_{0}\right)$ for the relevant AUG parameters: a parallel length of $L_{\|}=1.05 \mathrm{~m}$, a perpendicular radial length (along $x$ ) of the protruding limiters $L_{\perp}=0.012 \mathrm{~m}$, a plasma density in the private antenna region $n_{\mathrm{e}}=8 \cdot 10^{17} \mathrm{~m}^{-3}$ and an RF frequency of $36.5 \mathrm{MHz}$. Figure 2 describes the geometry of the calculations and the $3 \mathrm{D}$ weighting function $G_{3 \mathrm{D}}$, which is a 3D Green's function calculated according to [14]. The dimensions along $y$ (mostly poloidal) in the calculation frame are assumed to be infinite. The values of $G_{3 \mathrm{D}}$ imply integration along $y$ separately from the integration along the field line (z). A single point RF emitter is defined at $\Delta z=0$, $x=0$. The main feature of figure 2 is the strong parallel decay (along $z$ ) of the weighting factor. This is explained by the evanescent propagation of the slow wave at the frequency well above the lower hybrid frequency in the frame limited by the sheath boundary conditions, which strongly influences propagation. The function $G_{3 \mathrm{D}}$ experiences a decrease at a parallel distance of $\Delta z=0.5 \mathrm{~m}$ by more than a factor of 10, which corresponds to about half of the toroidal dimension of the 3-strap antenna. The transverse poloidal distribution (on the $x y$ plane) of the weighting function is about $1 \mathrm{~cm}$ broad and becomes more homogenous when the parallel distance is increased. This is explained by the coupling of the slow wave field between neighbouring magnetic field lines.

Thus, for the relevant AUG parameters the parallel decay of the slow wave field is strong. In this case, it is indeed reasonable to assume that the effect of the local $E_{\|}$-field close to the locations where the RF sheaths are formed is dominant for the RF sheaths. At the same time, the independent influence of the remote RF $E_{\|}$-field on local antenna-plasma interactions by the DC-biasing of the field lines due to the rectified sheaths on the opposite side of the antenna should be considered. This effect has been observed in many experiments in which the DC footprint of an active antenna was measured by a reciprocating probe several metres away from the antenna along the magnetic field lines [15, 16]. For the 3-strap antenna, the influence of one side of the antenna on the other due to this DC effect is lower than that for the 2-strap antenna. This is due to the fact that on both sides of the 3-strap antenna, the RF $E_{\|}$-field can be minimized simultaneously using the RF image current cancellation discussed next.

\subsection{RF image current cancellation and $E_{\|}$-field}

Figure 3 shows the principle of minimizing the RF sheaths at the limiters, and consequently minimizing $\mathrm{W}$ sputtering, by cancelling the RF image currents at the antenna limiters of the 3-strap antenna. The cases for a ratio of the power from the central strap to the power from the outer straps of $P_{\text {cen }} / P_{\text {out }}=0.1, P_{\text {cen }} / P_{\text {out }}=2$ and $P_{\text {cen }} / P_{\text {out }}=10$ are shown from left to right accordingly. At $P_{\text {cen }} / P_{\text {out }} \approx 2$, the RF image currents of these straps on the side limiters approximately cancel each other out. The cancellation is achieved on both the left- and right-hand sides of the antenna simultaneously, as opposed to the 2-strap antenna 


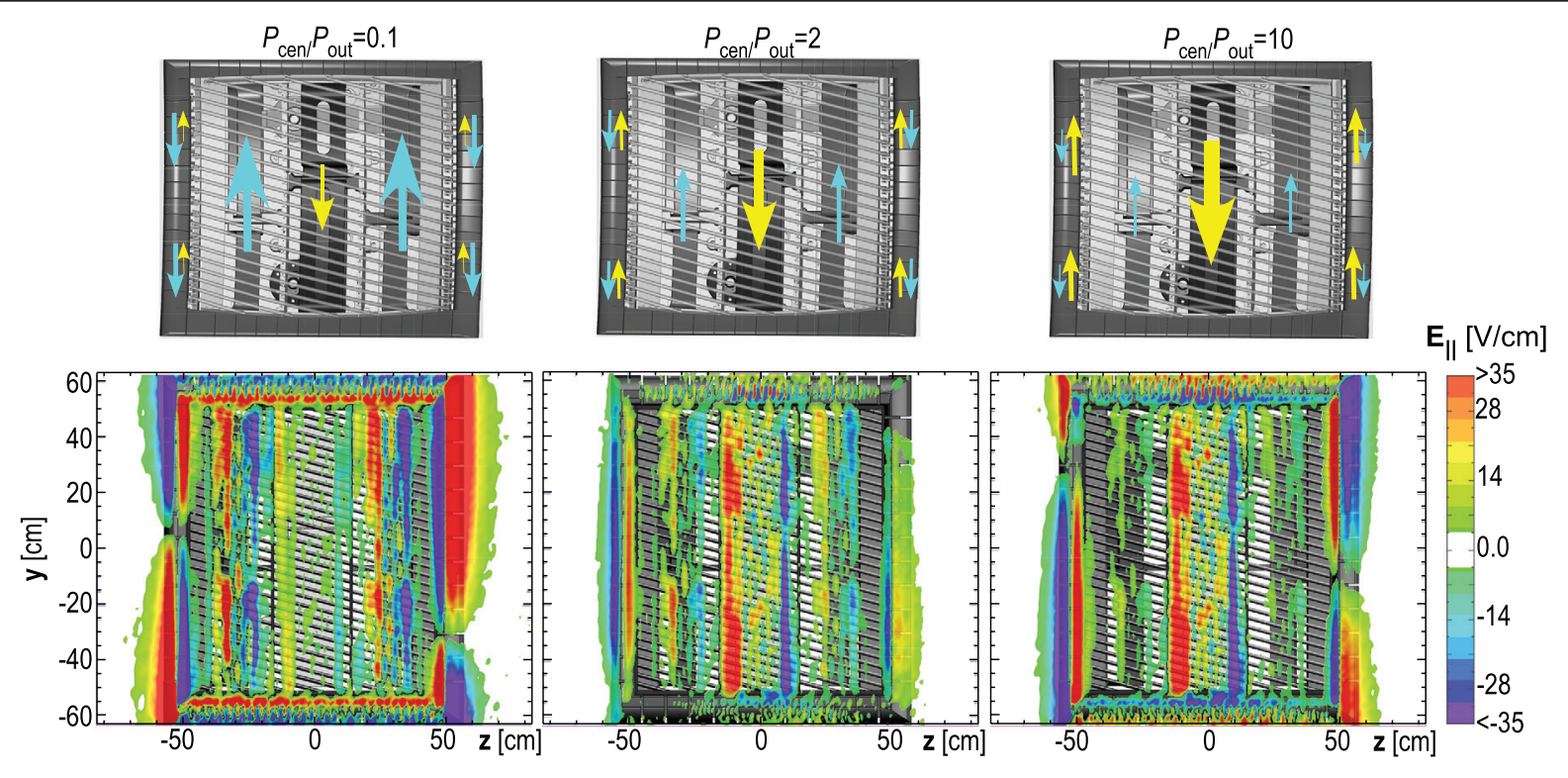

Figure 3. Upper row: the RF image current cancellation close to a power ratio of 2:1 (middle) in dipole phasing, compared to a power ratio of 1:10 (left) and 10:1 (right). The 3-strap antenna is shown using a CAD view with every second FS rod removed. Lower row: the corresponding TOPICA calculations of $\operatorname{Re}\left(E_{\|}\right)$at $36.5 \mathrm{MHz}, 0.5 \mathrm{MW}$ power, in a plane in front of a flat model of the antenna.

where cancellation is only possible on one side, as experimentally observed in [10].

The effect of image current cancellation on $E_{\|}$is shown in the lower part of figure 3 by the calculations of $\operatorname{Re}\left(E_{\|}\right)$ (the dominant part of the fields) in front of the antenna by the linear electromagnetic TOPICA code [17] for a flat AUG 3 -strap antenna model. The coordinate system for figure 3 is the same as in figure 2, except that in the former, $z$ corresponds to purely toroidal and $y$ corresponds to purely poloidal directions. The antenna aperture, the boundary between the vacuum region of the antenna and the plasma region described by the FELICE code [18] embedded in TOPICA, was set radially as close as possible to the antenna limiters, in order to minimize the filtering of small-scale RF fields. The plasma conditions and antenna settings correspond to AUG discharges \#32445 and \#32002 discussed below.

As implied in section 1.1, we consider the distribution of $E_{\|}$at the side limiters to be most important for the RF sheaths. The $E_{\|}$maps in figure 3 suggest that this is a reasonable assumption in all the cases shown: the field at the limiters is typically higher than the field close to the outer straps (closest to the limiters), and the field close to the central strap is too distant to have a strong effect on the RF sheaths on the side limiters, considering the parallel decay discussed above.

It is important to note that the reduction of $E_{\|}$by the cancellation of the RF image currents on the antenna side limiters is location dependent. In the case of dipole phasing and $P_{\text {cen }} / P_{\text {out }}=2$, values of $E_{\|}$close to zero are achieved at the upper and lower sections of the limiters. Compared to these regions, the regions around $y=0$ are characterized by a higher $E_{||}$-field for this case. Unique diagnostics, which take local measurements of the RF current at the limiters in the AUG, allow us to check whether similar behaviour is observed in the experiments and whether these RF currents do indeed correlate with the $\mathrm{W}$ sputtering.

\section{Experimental results and discussion}

We give a description of the experimental setup and diagnostics in section 2.1. In order to prove the relevance of the optimization approach used, in section 2.2 the measurements of the local RF currents are compared to the calculations of the relevant local RF quantities scanning the 3-strap antenna power balance. In section 2.3, the comparison is extended using strap phasing as an additional variable, and $\mathrm{W}$ sputtering is discussed. The possible reason for the differences between the experiment and the calculations is discussed in section 2.4. Section 2.5 is dedicated to the characterization of the total impurity production by the 3 -strap antenna and its reduction compared to the 2-strap antenna.

\subsection{Experimental setup}

Four ICRF antennas (al-a4, see figure 1), are used with a standard hydrogen-minority scheme in deuterium (D) or in helium (He). For this paper, data from the discharges with a toroidal magnetic field of $B_{\mathrm{t}}=2.5 \mathrm{~T}$ and a working frequency of $36.5 \mathrm{MHz}$ is used. Two 2-strap antennas, $a 1$ and $a 3$, constitute one antenna pair and they are powered by the 3 -dB hybrid scheme [19]. Antennas $a 2$ and $a 4$ have three straps which are powered using two 3-dB splitters, as shown in figure 4 .

Both $a 2$ and $a 4$ are equipped with local RF image current measurements arranged as shown in figure 4 , with six on the right-hand limiter of $a 2$ numerated as $a 2-1-a 2-6$ and four on each side of $a 4$, numerated $a 4-1-a 4-8$. At fixed limiter geometry and loading conditions (plasma profiles), the local amplitudes of the total RF electric field and of $E_{\|}$can be assumed to be directly proportional to the RF current measurements. Antenna a4 has spectroscopic spots of observation in locations $a 4-1, a 4-2, a 4-5$ and $a 4-6$ (see figure 1 for the toroidal and figure 4 for the poloidal locations), which measure the intensities of the W I, $400.9 \mathrm{~nm}$ and the D I, $410 \mathrm{~nm}$ spectral 


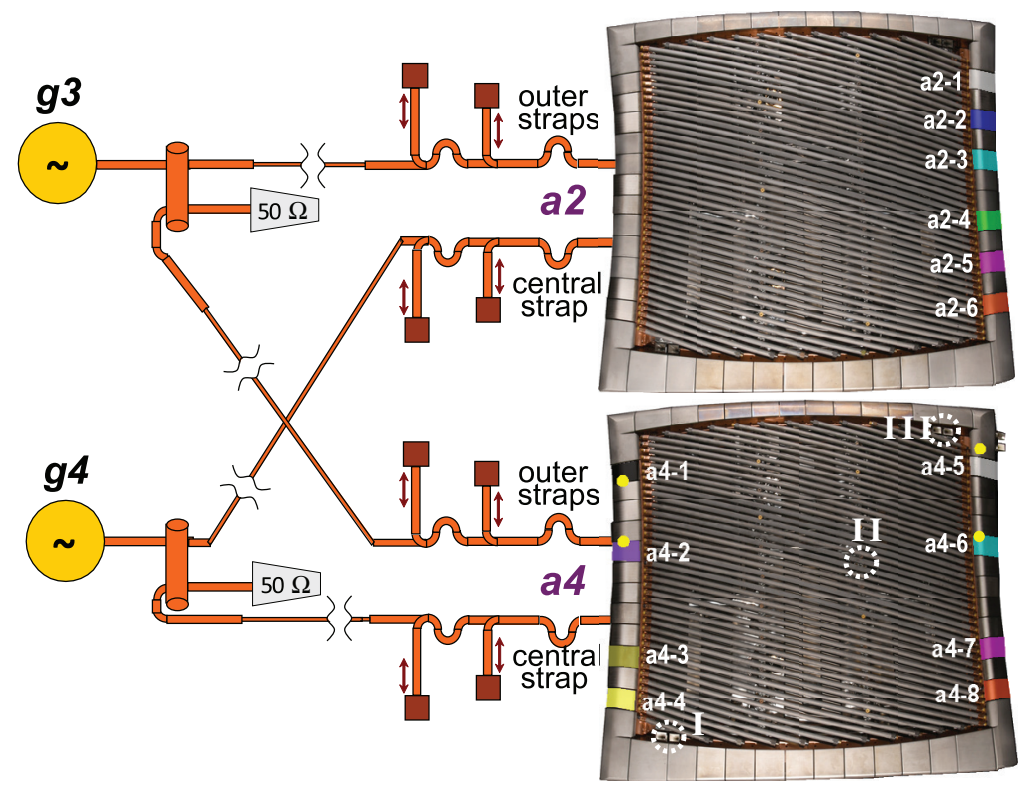

Figure 4. The 3-strap antenna connections; the local spectroscopy (yellow circles) and the RF current measurements are shown on the antenna limiters; the reflectometry locations $I-I I I$ are indicated.

a)

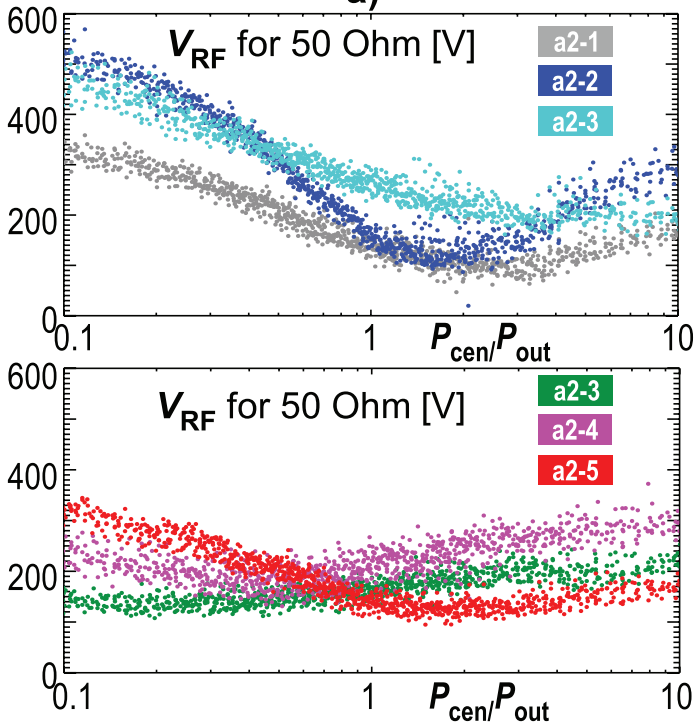

b)
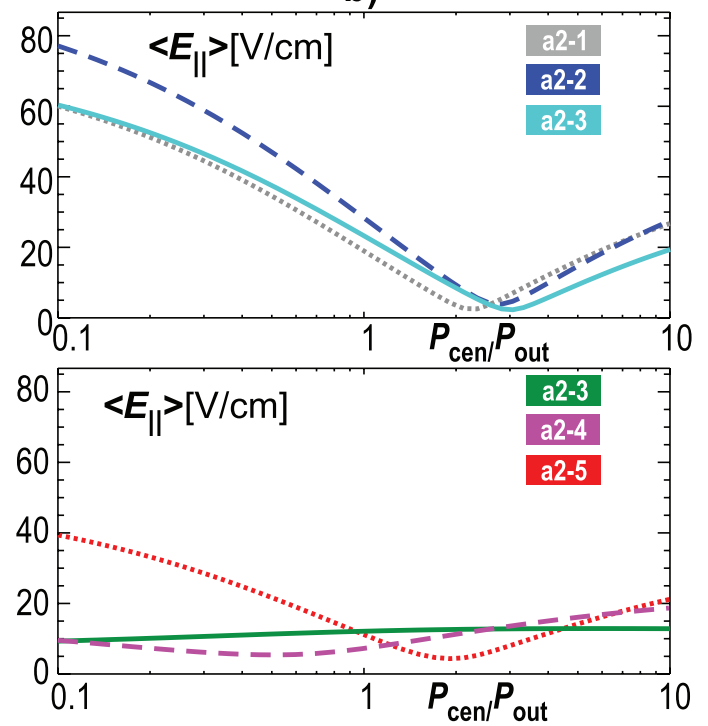

Figure 5. The dependency of the RF amplitudes on the $a 2$ antenna limiters as a function of the fraction of RF power from the central strap: (a) measured (5 ms averages); (b) calculated by TOPICA for the flat antenna model.

lines. The intensities are converted into $\mathrm{W}$ influx and the effective W sputtering yield. For three locations in front of $a 4$, indicated as $I, I I$ and $I I I$ in figure 4, reflectometry measurements are installed [20], which resolve the electron density profiles with a time resolution of $100 \mu$ s.

\subsection{Antenna power balance in dipole phasing}

The response of the RF current measurements at the limiters to a scan of the power balance between the central and outer straps of the dipole phasing for the D H-mode discharge $\# 32445, P_{\text {aux }}=5 \mathrm{MW}$, and an addition of the constant $P_{\mathrm{ICRF}}=1 \mathrm{MW}$ from both $a 2$ and $a 4$ during the scans is shown in figure 5(a). This discharge configuration allows the perturbation for the RF power to be relatively small. Thus, the influence of the non-linearities connected to the changes in the core plasma during the scans of the antenna feeding parameters is small. At the same time, the ELMs were mitigated using saddle coils [21] to provide quiet ELM-free conditions in the SOL. The RF voltage equivalent for a $50 \Omega$ load in a vacuum estimated from the RF current measurements is plotted as a function of $P_{\mathrm{cen}} / P_{\text {out }}$ for the six locations on the $a 2$ limiters. Every point corresponds to a time-average over $5 \mathrm{~ms}$. The experimental data in figure 5(a) is compared to figure 5(b) with the TOPICA calculations of the local $E_{\|}$-field averaged spatially over the corresponding locations of the limiters. The absolute values of the RF amplitudes in the experiment and in the calculations are not equivalent, because only the vacuum 
a)

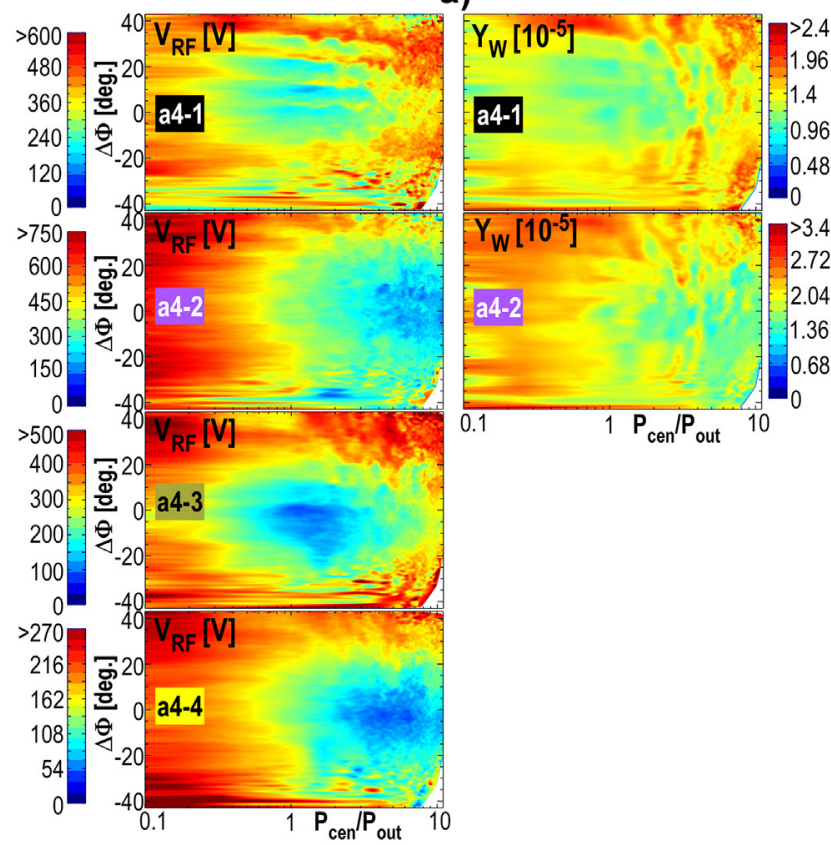

b)

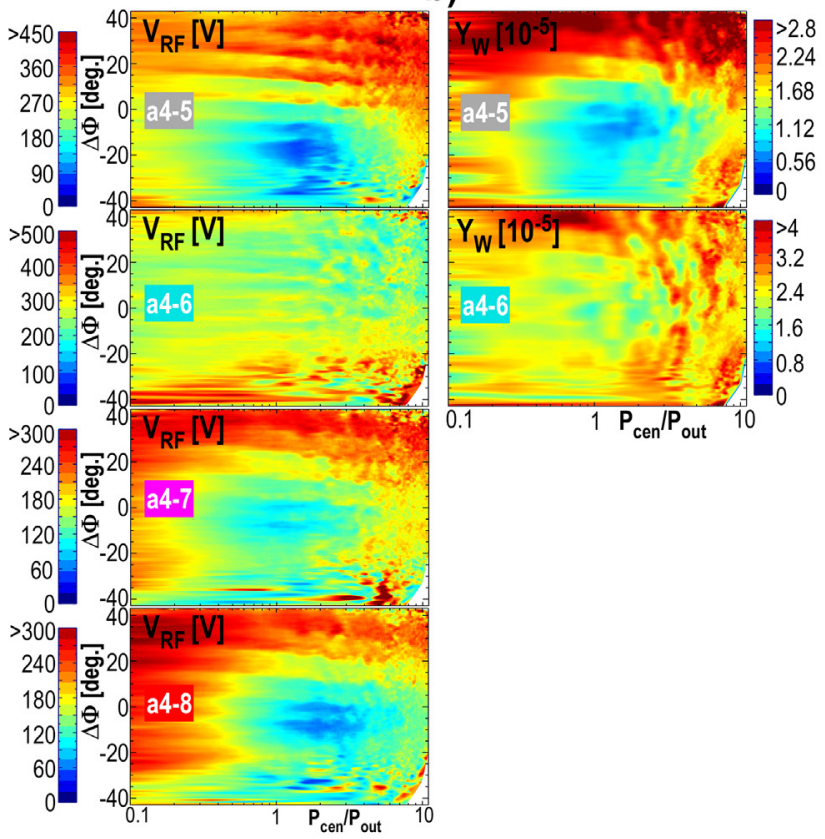

Figure 6. The RF voltage $V_{\mathrm{RF}}$ (equivalent to $50 \Omega$ in a vacuum) measured at the limiters of $a 4$ and the correlation to the W effective sputtering yield $Y_{\mathrm{W}}$ in the locations close to the RF measurements; (a) the left-hand limiter of a4, locations $a 4-1-a 4-4$; (b) the right-hand limiters of $a 4$ with locations $a 4-5-a 4-8$.

calibration can be done for the RF measurements, and this does not take into account the influence of plasma on the local RF circuit. Nevertheless, the relative behaviour can be compared. The measurements are well described by the local field magnitudes from the calculations - at least qualitatively. The tendency of the RF amplitude in the lower and the upper corners of the antenna (a2-1, a2-2, a2-5) to have a well-distinguished minimum is seen both in the measurements and in the calculations, as well as the tendency to have a flatter reaction to the power balance closer to the antenna middle ( $a 2-3, a 2-3$, a2-4). Thus, the local RF measurements at the limiters are represented well by the locally excited RF field calculated by TOPICA without taking into account slow wave propagation. However, we note that the measurements are less responsive to the power balance scan than suggested by the calculations. The possible reason for this is discussed in section 2.4.

\subsection{Phase-resolved amplitude balance and W sputtering}

A more sophisticated analysis than that in figure 5 can be made using both the scan of the power balance $P_{\text {cen }} / P_{\text {out }}$ and a scan of the phase deviation from the dipole $\Delta \Phi$. Figure 6 illustrates such 2D dependency of the RF amplitude on the limiters $V_{\mathrm{RF}}$ of $a 4$ when both the parameters were scanned simultaneously in discharge \#32002, which has the same conditions as \#32445. The plots in figure 6(a) correspond to the left $a 4$ limiter with locations from $a 4-1-a 4-4$ and the plots in figure 6(b) correspond to the right $a 4$ limiter with locations from $a 4-5-a 4-8$.

In addition to $V_{\mathrm{RF}}$, figure 6 presents the measurements of the effective $\mathrm{W}$ sputtering yield $Y_{\mathrm{W}}$ in locations $a 4-1, a 4-2$, $a 4-5$ and $a 4-6$ to the right of figures 6(a) and (b). Although the phase resolution of the data is limited, the minima of $V_{\mathrm{RF}}$ are visible, which also approximately translate into regions with reduced $Y_{\mathrm{W}}$. There is a good correlation between $V_{\mathrm{RF}}$ and $Y_{\mathrm{W}}$, although these two quantities are connected through highly non-linear mechanisms which involve RF sheath rectification and $\mathrm{W}$ sputtering mainly by the light impurity ions of carbon, boron, oxygen and nitrogen [2] with a certain distribution of concentrations and charge states. This provides a strong indication that in order to reduce $\mathrm{W}$ sputtering, one needs to reduce the local values of the RF image currents and thus the sheath-driving RF voltages. However, the DC effect of plasma biasing on the field lines connected to remote RF currentcarrying antenna components on $\mathrm{W}$ sputtering can also play a role. This effect could explain the slightly weaker reaction of $Y_{\mathrm{W}}$ to $V_{\mathrm{RF}}$ in locations $a 4-1, a 4-2, a 4-4$, which connect along the magnetic field lines to the limiter at the other side, and the stronger reaction in a4-3 without a connection to the other side limiter.

We note that the RF measurements on the limiters of the two 3-strap antennas $a 2$ and $a 4$ during the scans of the feeding parameters are not exactly the same. Antenna a2 usually shows a more sensitive response, although the main features of the dependencies remain similar. The reasons for this are likely to be related to the small differences in alignment of the internal antenna geometry and to the diversity of the surroundings in the AUG vacuum vessel.

The RF quantities from figure 6 can be compared to those of the TOPICA calculations discussed in section 1.2 for the dependence on the antenna power balance. The TOPICA results, now as functions of both $P_{\text {cen }} / P_{\text {out }}$ and $\Delta \Phi$, are presented in figure 7 for the same locations as in the experiments. The experimental measurements (figure 6) and the numerical results (figure 7) agree well regarding the existence of the minima of the RF quantities and on the shifts of the $P_{\text {cen }} / P_{\text {out }}$ values of these 

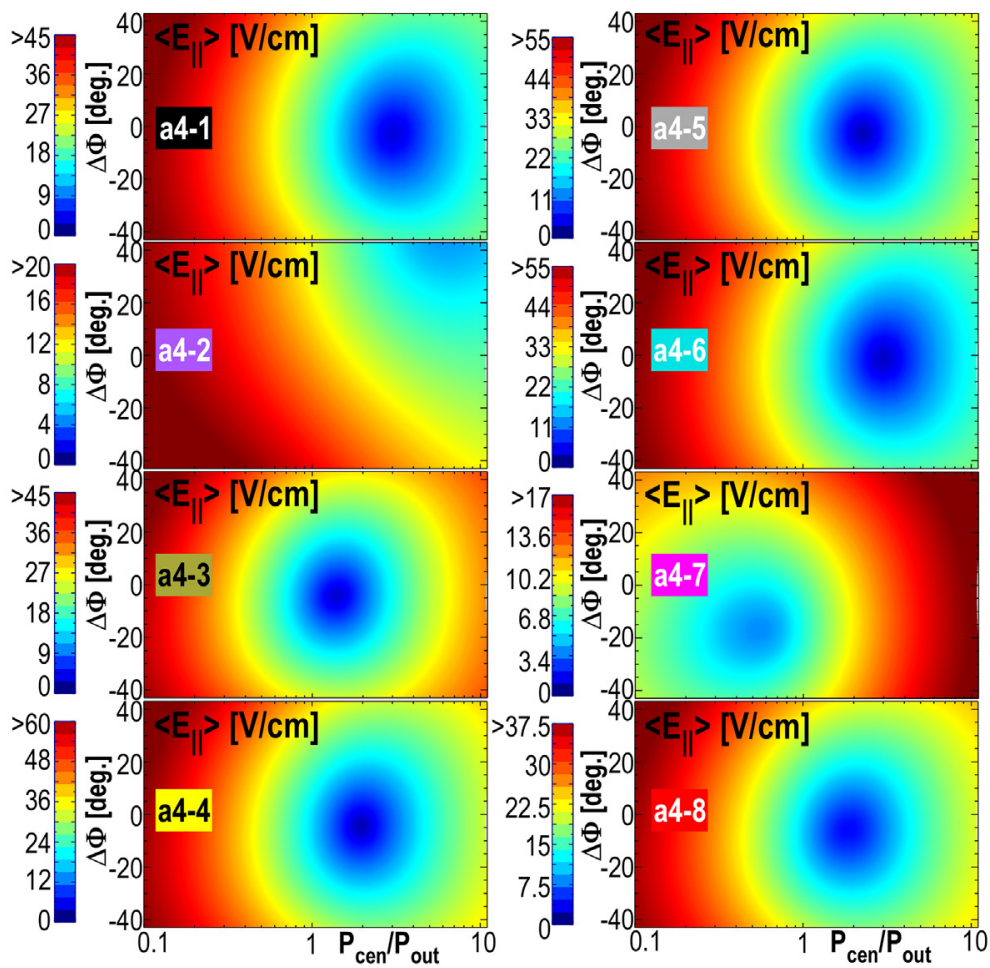

Figure 7. The TOPICA flat-model calculations of the spatially averaged $E_{\|}$as a function of $P_{\text {cen }} / P_{\text {out }}$ and $\Delta \Phi$ in the eight locations of the a4 side limiters, which correspond to the locations of the RF measurements in figures 5(a) (left column) and (b) (right column).

a)

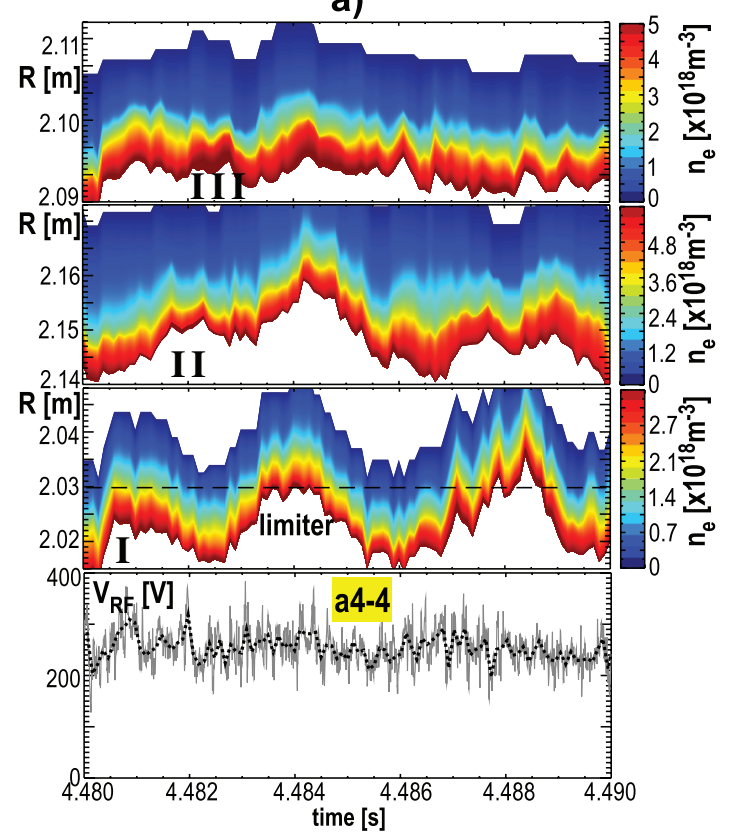

b)

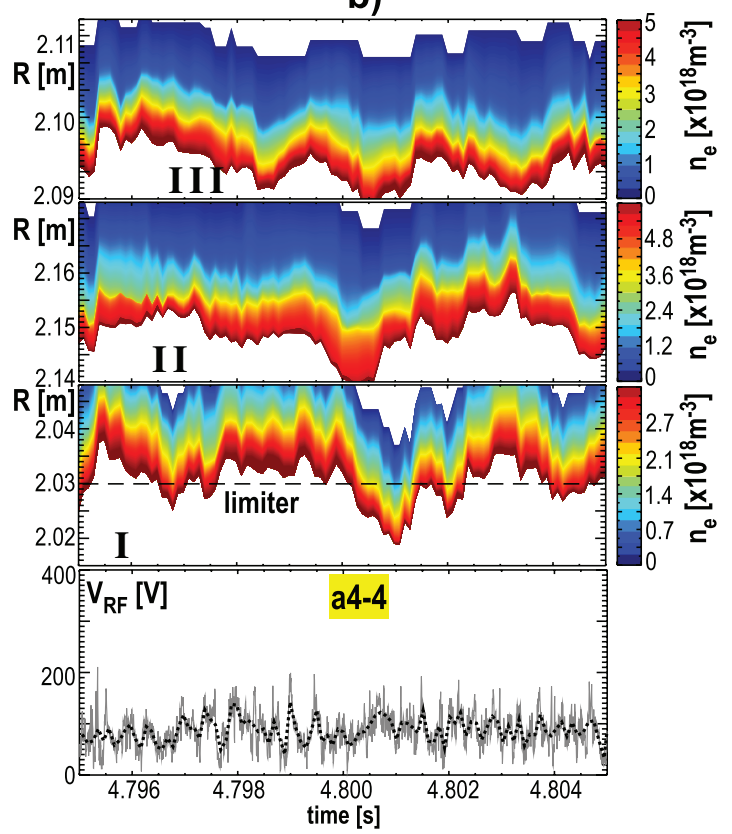

Figure 8. Upper three graphs: the electron density in front of $a 4$ in \#33336 in three locations from figure 3(b). Lower graphs: the RF amplitude in location $a 4-4$ with the signal averaged over $100 \mu$ s shown by the black dotted curve. (a) $P_{\text {cen }} / P_{\text {out }} \approx 0.1$; (b) $P_{\text {cen }} / P_{\text {out }} \approx 2.5$.

minima from location to location. The exact values of $P_{\text {cen }} / P_{\text {out }}$ and $\Delta \Phi$ for the minima show a less good agreement in some locations - especially in locations $a 4-2$ and $a 4-6$ where the qualitative behaviour of the dependencies experiences a change.

Similar to the data from figure 5 , the sensitivity of the $\mathrm{RF}$ response in the experiment is lower than that in the calculations. We hypothesize that apart from the limited phase resolution of the data and deviations of the real geometry from the modelling geometry of the antenna, two of the main reasons for this are the strong temporal and spatial perturbations of the density profile in front of the antennas which are present during plasma discharges. 


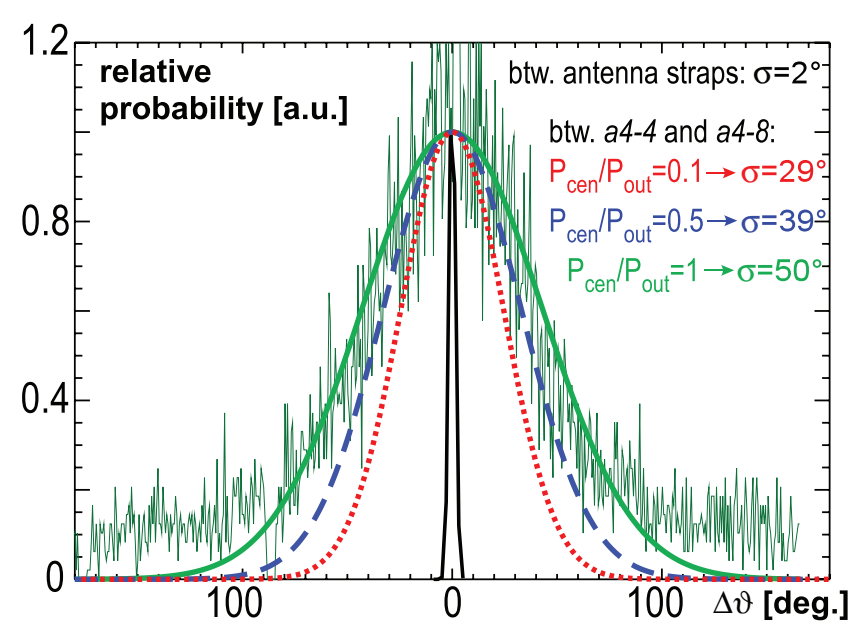

Figure 9. A histogram of the deviation of the phase between the a4 straps (black) and between the antenna limiters $a 4-4$ and $a 4-8$ for various $P_{\text {cen }} / P_{\text {out }}$. Raw data is shown for the case of $P_{\text {cen }} / P_{\text {out }} \approx 1$.

\subsection{Decorrelation of the RF image currents by density fluctuations}

The previous analysis used time-averaged values of RF amplitudes at the limiters from the experiment. However, the time-resolved signals show significant fluctuations, as is illustrated in the example of the RF amplitude in location a4-4 in figure 8 in the lower graphs. The imbalanced dipole case with $P_{\text {cen }} / P_{\text {out }} \approx 0.1$ is plotted in figure $8($ a) and the balanced dipole case with $P_{\text {cen }} / P_{\text {out }} \approx 2.5$ close to the balanced one is plotted in figure $8(\mathrm{~b})$. On the upper three graphs, both in figures 8(a) and (b), the plasma density profiles which are measured by antenna-embedded reflectometry at the three locations (I, II and $I I I)$ in front of $a 4$ are shown (see figure 4). The data is taken from discharge \#33336 with the same scenario as \#32445 and \#32002. For reflectometry location I, the radial position of the antenna limiter for $a 4-4$ is shown by a dashed line. Comparing the evolution of the density profiles in locations $I, I I$ and $I I I$, a high degree of spatial asymmetry is observed. This happens despite the fact that large ELMs are mitigated and only small intermittent events appear.

Figure 8 shows that the RF amplitude at $a 4-4$ which is close to location $I$, does not clearly correlate with the density variation in $I$, despite the large variations. This seems to confirm that the asymmetric density distribution across the antenna influences the RF image current balance both globally and locally. Fluctuations in the toroidal and poloidal density distributions can decorrelate the contributions of the central and the outer straps and make one of the contributions transiently stronger.

The density distribution and asymmetries are also affected by the convective cells due to the DC biasing of the field lines induced by the RF sheaths (see e.g. [22] and the references therein). Depending on the antenna design, antenna feeding and measurement location, the local density can either decrease or increase. An indication of this phenomenon, leading to a decrease in the density for the case of the imbalanced 3-strap antenna (figure 8(a)), is observed by reflectometry measuring in location $I$. Close to the radius of the antenna limiter, the density in figure 8 (a) $\left(P_{\text {cen }} / P_{\text {out }} \approx 0.1\right)$ is on average lower than in the balanced case in figure $8(\mathrm{~b})$

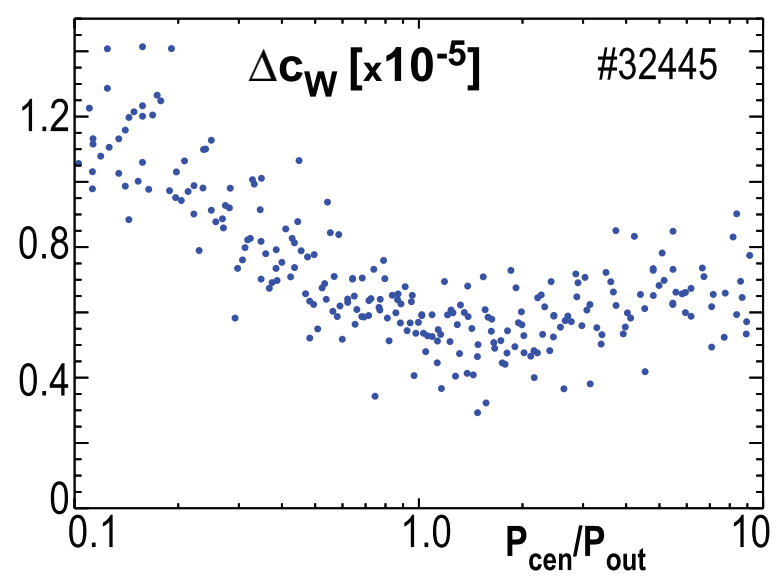

Figure 10. The influence of power balance in the dipole on the ICRF-specific W content in the core plasma (at $T_{\mathrm{e}} \approx 1.5 \mathrm{keV}$ ). Every point is a $20 \mathrm{~ms}$ average.

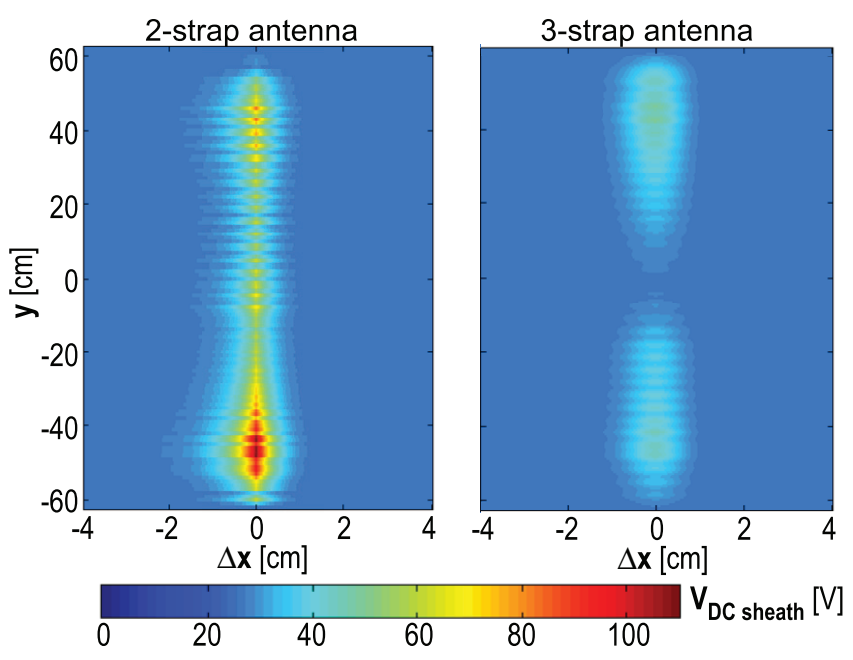

Figure 11. SSWICH-SW calculations of the $V_{\mathrm{DC}}$ sheath with flat models of the 2-strap (left) and 3-strap (right) antennas for $1 \mathrm{MW}$ per antenna.

$\left(P_{\text {cen }} / P_{\text {out }} \approx 2.5\right)$. This complicates the situation even further and can enhance the density asymmetries.

To understand better the mechanism leading to the observed modulation level of the fluctuations of the RF amplitude in figure 8 , we can use the independent information on the uncertainty of the RF phase measured between the antenna limiters a4-4 and a4-8. This uncertainty grows significantly when approaching the balanced case in figure 8(b), indicating a significant decorrelation of the RF image current contributions.

Histograms of the deviation $\Delta \vartheta$ of the RF phase are presented in figure 9. Despite the fact that the strap phasing is controlled within the small phase uncertainty of $\sigma=2^{\circ}$ ( $\sigma$ is the standard deviation in the Gaussian distribution), a relatively large phase uncertainty of $\sigma=29^{\circ}$ is observed on the antenna limiters, even when the contribution from the outer straps on the RF image current is dominant at $P_{\text {cen }} / P_{\text {out }} \approx 0.1$. When the power ratio is increased towards conditions of better RF local current cancellation, the intermittency of the plasma is accompanied by more even competition between the strap contributions to the local RF current. This translates into a higher phase uncertainty, with $\sigma=39^{\circ}$ for $P_{\text {cen }} / P_{\text {out }} \approx 0.5$ and with 
a)

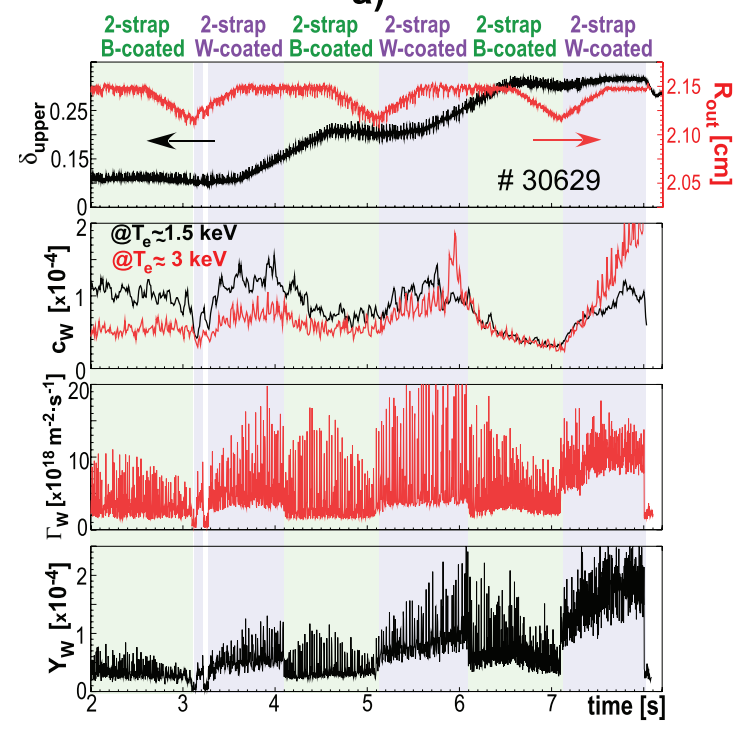

b)

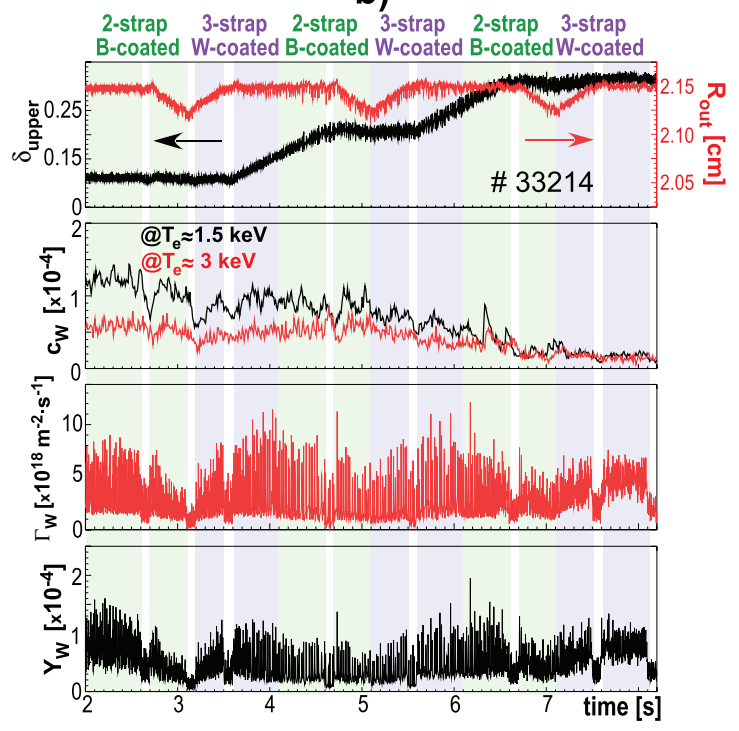

Figure 12. A comparison of the B-coated antennas with the W-coated antennas during scans of plasma triangularity and the radial position in deuterium. Every antenna pair $P_{\mathrm{ICRF}}=1.5 \mathrm{MW}$ in the highlighted time windows on top of $P_{\text {aux }}=6.3 \mathrm{MW}$. (a) The 2-strap antennas are W-coated (2014); (b) the 3-strap antennas are W-coated (since 2015, see figure 1).

$\sigma=50^{\circ}$ for $P_{\text {cen }} / P_{\text {out }} \approx 1$. For a power ratio of $P_{\text {cen }} / P_{\text {out }} \approx 2.5$ and higher, when the time-averaged local RF amplitude reaches a minimum, the phase uncertainty approaches $180^{\circ}$ (not shown in the figure), because a small perturbation of the density profile can define whether the contribution from the outer straps or the $180^{\circ}$-phased contribution of the central strap is stronger. With this large phase uncertainty the net RF current is still relatively small. However, zero cannot be reached on average, because the 3D density distribution needed for full RF current cancellation is very specific. This mechanism can also explain the fact that in sections 2.2 and 2.3 the TOPICA calculations for the homogenous plasma density profiles show higher sensitivity to the variation of the antenna feeding parameters than in the experiment.

The question arises: what can be done to reduce the effect of decorrelation of the RF image current contributions? Two general ways can be pointed out: (a) make the antenna design minimize the reaction of the RF image current distribution to density fluctuations; (b) decrease the degree of the density asymmetries. The first way has not been approached so far; the second way can be tackled by positioning the antenna differently in the fusion device. One of the ways of reducing the density asymmetries in the antennas is to align them with respect to the total magnetic field. This avoids the magnetic field lines, which are not seen by the whole array of the antenna straps, and should provide a more homogeneous density distribution in front of the antenna. As we know from [9], the field-aligned antenna in the Alcator C-Mod was characterized by a significant reduction in impurity sources during ICRF, with the local ICRF impurity sources effectively eliminated. However, it is not clear if this improvement is in some way connected to the decorrelation of the RF image currents. In the ASDEX Upgrade it is difficult to assess the role of the field inclination on the $\mathrm{W}$ sputtering and on the RF image current decorrelation, because a decrease in the magnetic field inclination angle is accompanied by an increase in density in the far SOL. The increased density reduces the $\mathrm{W}$ sputtering on its own and the corresponding change in density fluctuations complicates the analysis even more.

\subsection{Reduction of total W release}

The local minima of the RF current and of the $\mathrm{W}$ sputtering yield discussed in sections 2.2 and 2.3 contribute to a minimum of the total W source, although not all of the local minima appear at the same power ratio and phasing deviation from the dipole. Figure 10 shows the ICRF-specific change of the W concentration $\Delta c_{\mathrm{W}}$ measured close to $T_{\mathrm{e}}=1.5 \mathrm{keV}$ as a function of the strap power ratio $P_{\text {cen }} / P_{\text {out }}$ in the dipole phasing in \#32445 discussed in section 2.2. The minimum of the $\mathrm{W}$ content increment is observed for $P_{\text {cen }} / P_{\text {out }}$ between 1.0 and 3.0. This is consistent with the values of $P_{\text {cen }} / P_{\text {out }}$ expected from the minima of the local quantities which were in their turn consistent with the TOPICA calculations to a large extent (figure 6). Therefore, the local W source modulation is likely to be responsible for the minimum of the $\mathrm{W}$ content. This also means that the role of the modifications of the $k_{\|}$-spectrum during the scan of $P_{\text {cen }} / P_{\text {out }}$ [23] is likely to be less important. However, the $k_{\|}$-spectrum defines the RF power absorption and coupling-its modifications could affect the antennaplasma interactions and in general should not be neglected.

The $\mathrm{W}$ release of the 3-strap antenna at low $P_{\text {cen }} / P_{\text {out }}$ is generally higher than that for high $P_{\text {cen }} / P_{\text {out }}$. This is in qualitative agreement with figure 3 , which shows an overall higher $E_{\|}$-field at the limiters for the former case than for the latter. This can be explained by the larger effect of the outer straps on the field at the limiters and by the cross-coupling between the antenna straps which is taken into account in the TOPICA calculations. The configurations with high $P_{\text {cen }} / P_{\text {out }}$ induce RF currents in the outer straps keeping the current distribution on the straps more favourable for the lower $E_{\|}$-field at the limiters than in the configurations with low $P_{\text {cen }} / P_{\text {out }}$. 


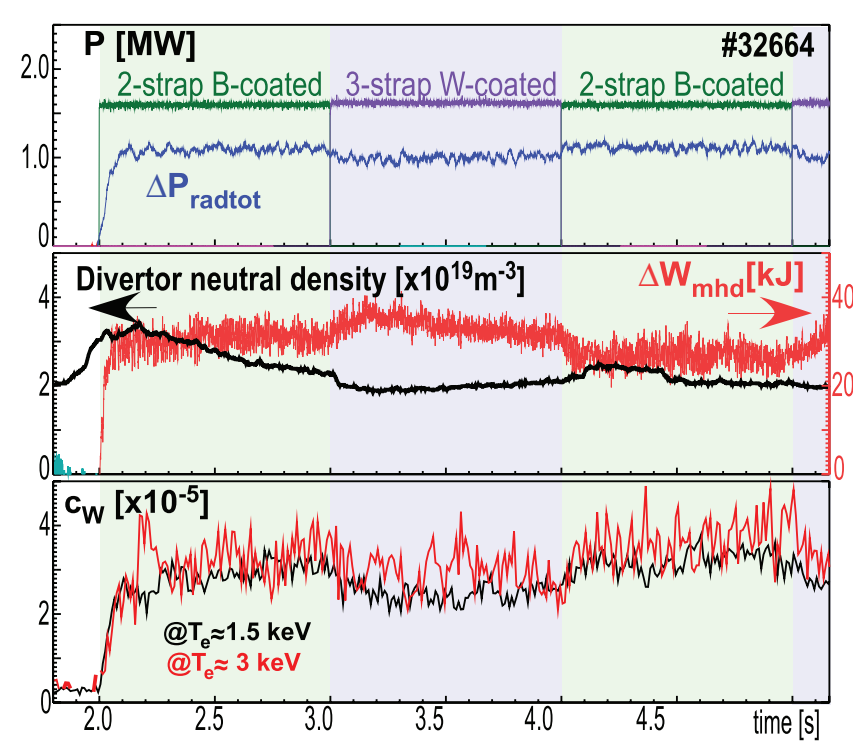

Figure 13. A comparison of the B-coated 2-strap antennas with the W-coated 3-strap antennas in the He-discharge \#32664.

A basic comparison between the 3-strap and the 2-strap antennas based on the electromagnetic calculations was done in [3]. In order to estimate a reduction in $\mathrm{W}$ production by the 3-strap antenna compared to the original 2-strap antenna, the rectified sheath potential $V_{\mathrm{DC}}$ sheath can now be estimated, as it is physically the closest electrical quantity to the $\mathrm{W}$ sputtering yield. This estimate is made by using the asymptotic version of the SSWICH-SW code [12]. It calculates $V_{\mathrm{DC}}$ sheath on the basis of the RF field maps in front of the antennas from the RAPLICASOL code [24], which allows the use of the latest version of SSWICH-SW. A homogenous density distribution in front of the antennas is used for the cases described in this paper. Figure 11 presents the calculations of the radial-poloidal ( $x y$ plane) distribution of $V_{\mathrm{DC}}$ sheath on the leading edge of the antenna side limiter using the radial distance from the leading edge $\Delta x$, both for the original 2-strap (left) and for the 3-strap (right) antenna with $P_{\text {cen }} / P_{\text {out }}=2.0$. The 3-strap antenna is characterized by a factor of about two to three reduced $V_{\text {DC sheath }}$ compared to the 2-strap antenna. This antenna improvement is roughly consistent with the experimental results which are presented in figure 12, where the antenna $\mathrm{W}$ release performance is compared relatively to the B-coated antennas in the D-discharges. The figure shows the $\mathrm{W}$ content in the core plasma as well as the $\mathrm{W}$ influx $\Gamma_{\mathrm{W}}$ and the effective W sputtering yield $Y_{\mathrm{W}}$ averaged over the measurements on the upper half of the right limiter of $a 4$ for the 2-strap antenna (2014, figure 12(a)) and the 3-strap antenna (since 2015, figure 12(b)) configurations, with details of the measurement locations described in [3, 25] correspondingly. A broad range of parameters is covered during the scans of the plasma triangularity $\delta_{\text {upper }}$ and the radial plasma position $R_{\text {out }}$ (see details on the effect of $\delta_{\text {upper }}$ and $R_{\text {out }}$ on ICRF-specific sputtering in [2, 26]) for $P_{\text {aux }}=5 \mathrm{MW}$ and $P_{\mathrm{ICRF}}=1.5 \mathrm{MW}$, with the latter toggling between the antenna pairs. The relative differences with respect to the B-coated antennas in figures 12(a) and (b) help to estimate the improvement of the W-coated 3-strap antennas compared to the W-coated 2-strap antennas. In the whole range of parameters, the use of W-coated 3-strap antennas significantly reduces $\mathrm{W}$ release. A reduction around a factor of two or higher of $\Gamma_{\mathrm{W}}$ and $Y_{\mathrm{W}}$ is observed, although these quantities are non-linear functions of $V_{\mathrm{DC}}$ sheath and should be compared to the latter with care.

It is interesting to look at the He-discharges, because these strongly expose the ICRF-specific $\mathrm{W}$ sputtering and provide further information on the distribution of the $\mathrm{W}$ sources. In $\mathrm{He}$, the W sputtering is amplified by a typically lower plasma density in the SOL and by the fact that $\mathrm{He}$ can sputter W directly within the range of ion energies in the SOL associated with the AUG ICRF operation [16]. A comparison of the W release between the 2-strap B-coated and the 3-strap W coated antennas in He characterized by $c_{\mathrm{W}}$ at $T_{\mathrm{e}} \approx 1.5 \mathrm{keV}$ and by the increment of the total radiated power $\Delta P_{\text {radtot }}$ is presented in figure 13 for discharge \#32664 with $P_{\text {aux }}=4.8 \mathrm{MW}$. The W release associated with the 3-strap antennas is lower and the energy content response of the plasma $\Delta W_{\text {mhd }}$ is higher. This stands in contrast with the D-operation for which the plasma energy response [23] and $c_{\mathrm{W}}$ (see [23] and above) are usually very similar for both pairs of antennas. One of the possible interpretations for this is that the remote $\mathrm{W}$ source (i.e. the $\mathrm{W}$ source which is not at the antenna limiters), which is presumably higher for the 2-strap antennas, becomes more important in the He-discharges. The increase in density of neutrals in the divertor shown in figure 13 when the 2-strap antennas are active would be consistent with stronger plasma-wall interactions leading to outgassing.

Finally, one has to note that the improvement in the 3-strap antennas comes at the price of having a maximum RF voltage which is about $20 \%$ higher in the antenna transmission lines compared to the 2-strap antenna-although the radiating area did increase by about $20 \%$. Moreover, the distribution of the RF power between the RF generators is uneven because of the imposed $P_{\text {cen }} / P_{\text {out }}>1.0$. The addition of another RF generator to the circuit of the central straps, planned for implementation in the ASDEX Upgrade, will provide the possibility of using all RF generators at full power.

\section{Summary and perspectives}

The agreement between the RF measurements at the antenna limiters in ASDEX Upgrade and the TOPICA simulations points to the local E-field at the limiters being one of the main drivers of the RF sheaths. This is the case when the slow wave is evanescent and experiences a strong decay along parallel distances smaller than the characteristic parallel dimensions of the antenna. Using the antenna power balance ratio and the strap phasing as variables for the 3-strap antenna, the local minima of the RF current at the limiters and of the W sputtering yield can be achieved in various locations of the antenna limiters. These minima do not always align with each other at the same values of the variables. Designing an antenna with location-independent minima in a broad range of loading conditions is challenging. An additional complication is the uncertainty of reaching these minima imposed by the intermittent density profile fluctuations and asymmetries, 
which are also non-linearly linked to local ICRF-induced density convection. Nevertheless, the 3-strap antenna with the W-coated limiters showed a significant reduction in $\mathrm{W}$ release compared to the $\mathrm{W}$-coated 2 -strap antennas in a broad range of plasma conditions. This reduction is approximately consistent with the non-linear estimates of the rectified sheath voltage in front of the antennas by the asymptotic SSWICH-SW code, which currently only takes slow wave propagation in the SOL into account. In the future, a fullwave SSWICH code should be able to implement SOL fast wave propagation close to the antenna [27] and model the RF near-fields more accurately.

Thus, in order to reduce the plasma-wall interactions close to the antenna in future ICRF antenna designs, the $E$-field needs to be reduced primarily on the radially protruding structures where the RF sheaths can form. This implies the minimization of the RF currents on such structures. As a matter of fact, this can also mean a reduction of the antenna power launched per area, as in the case of the 3-strap antenna.

For a more realistic plasma description in the electromagnetic and the non-linear calculations, an evolving 3D density distribution has to be implemented into the calculations. This should allow us to estimate the resilience of the RF image current balance of an antenna to density asymmetries and fluctuations.

As has been estimated in the past [23], the local W source from the antenna limiters, mostly affected by the antenna RF near-fields, was responsible for about $2 / 3$ of the $\mathrm{W}$ content in the plasma core. Once this has been eliminated by the boron coatings on the limiters and dramatically decreased by the 3 -strap antennas, the remote $\mathrm{W}$ source and the far-field effects become increasingly more important. Thus, an area of future study should include the minimization of remote $\mathrm{W}$ sources by affecting the global RF field structure [28] in experiments using such actuators as phasing between the antenna pairs, for example.

\section{Acknowledgments}

This work has been carried out within the framework of the EUROfusion Consortium and has received funding from the EURATOM research and training programme 2014-2018 under grant agreement No. 633053. The views and opinions expressed herein do not necessarily reflect those of the European Commission.

\section{References}

[1] Neu R et al 2007 Plasma Phys. Control. Fusion 49 B59-70

[2] Bobkov V et al 2010 Nucl. Fusion 50035004

[3] Bobkov V et al 2013 Nucl. Fusion 53093018

[4] Perkins F 1989 Nucl. Fusion 29583

[5] D’ippolito D A et al 1998 Nucl. Fusion 381543

[6] Mendes A et al 2010 Nucl. Fusion 50025021

[7] Garrett M L et al 2012 Fusion Eng. Des. 87 1570-5

[8] Colas L et al 2013 J. Nucl. Mater. 438 S330-3

[9] Wukitch S et al 2013 Phys. Plasmas 20056117

[10] Bobkov V et al 2015 AIP Conf. Proc. 1689030004

[11] Bobkov V et al 2009 AIP Conf. Proc. 1187125

[12] Colas L et al 2012 Phys. Plasmas 19092505

[13] Jacquot J et al 2014 Phys. Plasmas 21061509

[14] Colas L et al 2016 Plasma Phys. Control. Fusion in press

[15] Kubič M et al 2013 J. Nucl. Mater. 438 S509-12

[16] Colas L et al 2014 AIP Conf. Proc. 1580 259-62

[17] Lancellotti V et al 2006 Nucl. Fusion 46 S476

[18] Brambilla V 1993 Plasma Phys. Control. Fusion 3541

[19] Goulding R H et al 1996 AIP Conf. Proc. 355 397-400

[20] Aguiam D et al 2016 Rev. Sci. Instrum. 87 11E722

[21] Suttrop W et al 2011 Phys. Rev. Lett. 106225004

[22] Zhang W et al 2016 Plasma Phys. Control. Fusion 58095005

[23] Bobkov V et al 2016 Nucl. Fusion 56084001

[24] Jacquot J et al 2015 AIP Conf. Proc. 1689050009

[25] Bobkov V et al 2016 Nucl. Mater. Energy submitted

[26] Bobkov V et al 2007 J. Nucl. Mater. 363-5 122-6

[27] Lu L et al 2016 43rd EPS Conference on Plasma Physics, Leuven, Belgium, ECA 40A P2.068

[28] Ochoukov R et al 2016 Rev. Sci. Instrum. 87 11D301 